\title{
DESEMPENHO DE QUATRO ESPÉCIES DE Eucalyptus spp EM PLANTIOS PUROS E CONSORCIADOS COM SABIÁ (Mimosa caesalpiniaefolia Benth) EM CAVA DE EXTRAÇÃO DE ARGILA1 ${ }^{1}$
}

\author{
Andrea Vita Reis Mendonça², José Geraldo de Araujo Carneiro³, Deborah Guerra Barroso ${ }^{3}$, Anderson \\ Ribeiro Santiago ${ }^{4}$, Teresa Aparecida Soares de Freitas ${ }^{5}$, Josival Santos Souza ${ }^{2}$
}

\begin{abstract}
RESUMO - Para a recuperação ambiental de áreas mineradas, uma das alternativas é o florestamento ou reflorestamento, havendo a necessidade de selecionar espécies e práticas silviculturais que favoreçam o estabelecimento dos povoamentos. O objetivo deste trabalho foi avaliar o desempenho de Eucalyptus camaldulensis Dehnh, E. pellita $\mathrm{F}$. Muel, E. tereticornis $\mathrm{Sm}$. e E. robusta $\mathrm{Sm}$., em plantios puros e consorciados com a leguminosa arbórea Mimosa caesalpiniaefolia Benth, em áreas degradadas pela extração de argila. Este estudo foi realizado em uma cava de 1,5 ha, com solo salino, localizada em Campos dos Goytacazes, RJ, de onde foi removido argila até a profundidade média de $1,5 \mathrm{~m}$. O delineamento experimental foi em blocos ao acaso com quatro repetições, em esquema fatorial 4 x 2, com quatro espécies de eucalipto, em plantios puros, e cada uma delas em consórcio com M. caesalpiniaefolia . O desempenho das plantas foi avaliado quanto à sobrevivência, altura, diâmetro ao nível do solo (DAS), diâmetro a 1,30 m de altura (DAP), área de copa, razão altura/DAP, área superficial e comprimento de raízes finas (? $2 \mathrm{~mm}$ ). Até 24 meses, observou-se que E. pellita não é indicado para plantios em cavas de extração de argila, enquanto E. camaldulensis, E. tereticornis e E. robusta apresentam melhor desempenho. Não foi detectado, até o momento, efeito do sistema de plantio sobre o crescimento das quatro espécies de eucalipto. No entanto, foi verificado que o consórcio com sabiá resultou em efeito negativo sobre a sobrevivência do E. robusta. O consórcio entre essas duas espécies não é indicado para as condições deste experimento.
\end{abstract}

Palavras-chave: Eucalipto, plantio misto, raízes finas, área degradada, crescimento de mudas.

\section{PERFORMANCE OF FOUR SPECIES OF Eucalyptus PURE AND MIXED WITH SABIÁ (Mimosa caesalpiniaefolia Benth) IN CLAY EXTRACTION SITE}

\begin{abstract}
An alternative for environmental rehabilitation of areas degraded by clay extraction is reforestation with selected species and silvicultural practices to promote the establishment of stands. The objective of this study was the evaluation of the performance of Eucalyptus camaldulensis Dehen, E. pellita F. Muel, E. tereticornis Sm and E. robusta Sm in pure stands and mixed with "sabiá" (Mimosa caesalpiniifolia, Benth). This study was carried out on a dug area of 1.5 ha, saline soil, with average depth of $1.5 \mathrm{~m}$, in Campos dos Goytacazes, RJ. The experiment was arranged in randomized blocks with four replications, in a factorial design with four eucalyptus species in pure stands and mixed with "sabiá". Plant performance was evaluated in terms of survival percentage, height $(H)$, ground level diameter $(G L D)$, breast height diameter $(B H D), H / B H D$ ratio, surface area and fine
\end{abstract}

\footnotetext{
${ }^{1}$ Recebido em 19.03.2007 e aceito para publicação em 19.05.2008.

${ }^{2}$ Departamento de Engenharia Florestal da Universidade Federal do Recôncavo da Bahia (UFRB) - Cruz das Almas-BA. E-mail: <avrmendonca@ hotmail.com>.

${ }^{3}$ Universidade Estadual do Norte Fluminense, Campos dos Goytacazes-RJ. E-mail: <carneiro@uenf.br>; <deborah@uenf.br>.

${ }^{4}$ Instituto Natureza do Tocantins, Araguaína-TO. E-mail: <asantiago@ naturantins.to.gov.br>.

${ }^{5}$ Programa de Pós-Graduação em Produção Vegetal da UENF, Campos dos Goytacazes-RJ. E-mail: <tfreitas@uenf.br>.
} 
root length ( $2 \mathrm{~mm}$ ). Twenty-four months after planting, E. pellita was proven unsuitable for planting in dug areas. The planting system did not affect the growth of the eucalyptus species. However, the stands mixed with "sabia" showed a negative effect on E. robusta survival. Mixed planting of these two species is not indicated for the conditions of this experiment.

Keywords:Eucalyptus, mixed plantings, fine roots, degraded area and seedling growth.

\section{INTRODUÇÃO}

As atividades relacionadas à cerâmica são estratégicas para o desenvolvimento da região Norte fluminense, onde existem mais de 100 cerâmicas, que geram uma receita anual de cerca de $\mathrm{R} \$ 170$ milhões empregos diretos (RAMOS et al., 2003).

No diagnóstico realizado por Ramos et al. (2003) foi registrado que 83 cerâmicas consumiam um total de $5.700 \mathrm{~m}^{3} \mathrm{dia}^{-1}$ de argila. O método de extração consiste na retirada do solo com retroescavadeira, em profundidades variando de 1 a $4 \mathrm{~m}$ (VALICHESKI, 2004), embora a Fundação Estadual de Engenharia do Meio Ambiente (FEEMA) admita escavações com profundidade máxima de 2,5 m, respeitando-se o limite de afloramento do lençol. A área é profundamente alterada, e a Constituição Federal (Artigo 225, Parágrafo $2^{\circ}$ ) determina que o explorador recupere o ambiente degradado.

As cavas de extração de argila do Norte fluminense têm sido muito utilizadas para plantios de cana-deaçúcar e pastagem. Essas atividades, entretanto, envolvem manejo intensivo do solo, uso do fogo e muita movimentação de animais ou implementos agrícolas, técnicas convencionais que não contribuem para a recuperação ambiental da área. O plantio de espécies florestais de uso múltiplo que auxiliem a melhoria química e física da área pela deposição de serrapilheira, proporcionada pelo maior ciclo de produção, é uma possibilidade que pode permitir conciliar retorno econômico e sustentabilidade do empreendimento. Ressalta-se que a análise econômica de Eucalyptus camaldulensis, cana-de-açúcar e pastagem nessas cavas apontou a eucaliptocultura como atividade mais promissora (VALICHESKI, 2004). O florestamento poderá atender à demanda das indústrias de cerâmica, que têm enfrentado dificuldades na obtenção de lenha para o processo de produção, bem como fornecer outros produtos madeireiros.

No desenvolvimento de estratégias de recuperação ambiental que se baseiam na utilização de vegetais, deve-se dar especial atenção à escolha de espécies. Estas precisam ser tolerantes às situações adversas, como afloramento de lençol, solos com elevados teores de sódio trocável e com alta condutividade elétrica, condições freqüentemente encontradas na região de estudo e agravadas pela prática de exploração de argila (RAMOS e MANZATTO, 2003; VALICHESKI, 2004). O gênero Eucalyptus tem potencial para ser testado nessas áreas, pois, além de ter a aceitação dos produtores, apresenta crescimento rápido. Entretanto, não se conhece o comportamento de suas espécies nesses sítios.

A utilização de leguminosas fixadoras de nitrogênio consorciadas com eucalipto pode melhorar as condições edáficas e aumentar, eventualmente, a produção total dos povoamentos. Plantios consorciados podem proporcionar melhorias na estrutura do solo, aumentar o teor de matéria orgânica e a disponibilidade de nutrientes, além de promover condições ecofisiológicas favoráveis ao crescimento das árvores (GAMA-RODRIGUES, 1997). A tendência do favorecimento do crescimento e produção em plantios consorciados é evidenciada emEucalyptus camaldulensis com Acacia mangium (SCHIAVO et al., 2004), E. globulus com A. mearnsii (FORRESTER et al., 2004, 2005), E. saligna com Falcataria molucna (BINKLEY et al., 2003), Terminalia amazonica com Inga edulis (NICHOLS et al., 2001), Eucalyptus globulus com Acacia mangium (BAUHUS et al., 2000), Pinus taeda com Robinia pseudoacia (GRONINGER et al., 1997) e Tectona grandis consorciada com Leucaena leucocephala (KUMAR et al., 1998).

O manejo e a seleção de espécies são aspectos importantes em sistemas consorciados, portanto, se negligenciados, podem comprometer a produtividade desses sistemas, a exemplo de resultados insatisfatórios observados no consórcio entre Acacia dealbata e $E$. nitens (HUNT et al., 1999) e entre Eucalyptus urophylla e Leucaena leucocephala (JESUS e DIAS, 1988).

O objetivo deste trabalho foi avaliar o desempenho de Eucalyptus camaldulensis Dehnh, E. pellita F. Muel, E. tereticornis $\mathrm{Sm}$. e E. robusta $\mathrm{Sm}$. em plantios puros 
e consorciados com a leguminosa arbórea Mimosa caesalpiniaefolia Benth (sabiá), em áreas degradadas pela extração de argila.

\section{MATERIAL E MÉTODOS}

Este estudo foi realizado em uma cava de 1,5 ha, no Município de Campos dos Goytacazes, Rio de Janeiro ( $21^{10} 5^{\prime} \mathrm{S}, 41^{\circ} 14^{\prime} \mathrm{W}$, e altitude de $\left.11 \mathrm{~m}\right)$. Antes da exploração da argila, ocorrida entre janeiro de 1997 e agosto de 1998, a área estava sendo utilizada para plantio de canade-açúcar. A profundidade da cava ficou em torno de $1,5 \mathrm{~m}$.

Pela classificação de Köppen, o clima da região é tropical chuvoso (AW). Segundo a Empresa de Pesquisa Agropecuária do Estado do Rio de Janeiro (dados não publicados), a temperatura média mensal do município variou nos últimos 10 anos (1996/2006) entre $21,4^{\circ} \mathrm{C}$ e $27,7^{\circ} \mathrm{C}$, e a precipitação média anual foi de $852 \mathrm{~mm}$, com chuvas concentradas no período de outubro a dezembro.

O solo original da área da cava em estudo é um Cambissolo Háplico Sódico Gleico Salino, com profundidade de lençol de 3,2 m (VALICHESKI, 2004). O solo da cava, na ocasião da implantação do experimento, apresentou na camada de 0-20 cm: 8,9 $\pm 0,95 \mathrm{dS} \mathrm{m}^{-1}$ de condutividade elétrica, 5,97 $\pm 0,03$ de $\mathrm{pH}, 2,6258,5$ $\pm 8,19 \mathrm{mg} \mathrm{kg}^{-1} \mathrm{de} \mathrm{P}, 2,62 \pm 0,14 \mathrm{de} \mathrm{H}+\mathrm{Al}, 1,77 \pm 0,04$ de $\% \mathrm{C}, 7,21 \pm 0,38 \mathrm{cmol}_{\mathrm{c}} \mathrm{dm}^{-3} \mathrm{de} \mathrm{Ca}, 4,94 \pm 0,38 \mathrm{cmol}_{\mathrm{c}}$

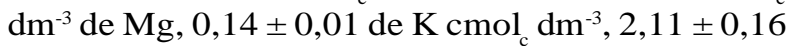
de $\mathrm{Na} \mathrm{cmol}_{\mathrm{c}} \mathrm{dm}^{-3}, 40,55 \pm 1,54 \mathrm{de} \%$ de argila, 27,39 $\pm 2,54$ de $\%$ de areia, $32,06 \pm 1,43 \%$ de silte, $1,24 \pm$ $0,02 \mathrm{Mg} \mathrm{m}^{-3}$ de densidade, $0,49 \pm 0,01 \mathrm{~m}^{3} \mathrm{~m}^{-3}$ de poros totais, $0,42 \pm 0,01 \mathrm{~m}^{3} \mathrm{~m}^{-3}$ de microporos e $0,08 \pm 0,01 \mathrm{~m}^{3}$ $\mathrm{m}^{-3}$ de macroporos (MENDONÇA, 2006).

As sementes de sabiá foram obtidas no CNPABEmbrapa (Seropédica, RJ) e as de eucalipto, no Instituto de Pesquisas e Estudos Florestais (IPEF). As mudas de eucalipto foram produzidas em tubetes de $50 \mathrm{~cm}^{3}$ e as de sabiá, em tubetes de $288 \mathrm{~cm}^{3}$. O substrato utilizado foi uma mistura de casca de eucalipto compostada e casca de arroz carbonizada, na proporção de 7:3 em volume, enriquecidos com osmocote 14-14-14 $\left(6 \mathrm{~g} \mathrm{~kg}^{1}\right)$. As sementes de sabiá foram inoculadas com rizóbio específico à espécie leguminosa (BR3405/BR3451), obtido no CNPAB-Embrapa (Seropédica, RJ).

Na ocasião da extração da argila, a camada superficial $(20 \mathrm{~cm})$ foi acumulada e, posteriormente, distribuída na cava. $\mathrm{O}$ solo foi gradeado e o plantio, realizado em covas de $20 \mathrm{~cm}$ de profundidade, no espaçamento 3 $2 \mathrm{~m}$, não sendo realizadas adubações de plantio e de cobertura.

Os tratos culturais realizados foram: controle de formigas com iscas granuladas; irrigação até o segundo mês de plantio; coroamento das mudas até o primeiro ano de plantio; podas das plantas de sabiá, aos 12 e 24 meses, mantendo de uma a três hastes por planta, sendo o material resultante da poda distribuído na linha de plantio dentro da parcela.

O delineamento experimental foi em blocos ao acaso, com quatro repetições, em esquema fatorial $4 \times 2$, com quatro espécies de eucalipto (Eucalyptus camaldulensis Dehnh, E. pellita F. Muel, E. tereticornis Sm. e E. robusta $\mathrm{Sm}$.), em plantios puros, e cada uma delas em consórcio com sabiá. Cada parcela foi composta por 36 plantas, dispostas em seis linhas. Nas parcelas consorciadas, as mudas foram plantadas em linhas alternadas, sendo as linhas pares compostas por eucalipto e as ímpares, por sabiá.

As plantas de eucalipto foram avaliadas quanto a sobrevivência, altura e diâmetro ao nível do solo (DAS), aos 3, 6, 9, 12 e 24 meses. As áreas da projeção da copa foram mensuradas aos 6,9 e 12 meses e o diâmetro a 1,30 m de altura (DAP), aos 24 meses. A mensuração das características biométricas foi realizada nas linhas pares, totalizando 18 plantas por parcela.

Aos 24 meses de idade, foram avaliados a área superficial e o comprimento de raízes finas $(\leq 2 \mathrm{~mm})$, nos dois sistemas de cultivo. As coletas foram realizadas com o auxílio de um trado $(\phi=2,5 \mathrm{~cm})$, na profundidade de 0 a $10 \mathrm{~cm}$. Em cada repetição foram tomadas duas amostras, retiradas a $20 \mathrm{~cm}$ de distância das plantas de altura média, na linha de plantio do eucalipto. As amostras foram acondicionadas em sacolas plásticas e armazenadas em "freezer". As raízes foram separadas do solo e colocadas, aleatoriamente, entre duas placas de vidro de $2 \mathrm{~mm}$ de espessura, $21 \mathrm{~cm}$ de largura e $30 \mathrm{~cm}$ de comprimento. Em seguida, obteve-se imagem pelo "scanner", sendo esta processada por meio do programa QuantRoot, desenvolvido pelo Departamento de Solos da Universidade Federal de Viçosa, o qual determina comprimento, área e diâmetro de raízes.

As plantas de sabiá foram avaliadas quanto a sobrevivência, altura e diâmetro do caule ao nível do solo (DAS), aos 24 meses.

R. Árvore, Viçosa-MG, v.32, n.3, p.395-405, 2008 
Os dados foram submetidos à análise de variância e ao teste Tukey de médias $(\alpha=0,05)$. Para o incremento em Altura e DAS avaliado ao longo do tempo, foi realizada uma análise de variância em parcelas divididas no tempo, considerando-se que a época foi um tratamento não passível de aleatorização (BANZATTO e KRONKA, 1992). O comportamento do crescimento em altura e DAS ao longo do tempo foram analisados por regressão sequiencial. Para verificar a validade dos modelos ajustados por regressão, foi realizado o teste de Durbin-Watson (CHATTERJU e PRICE, 1977), bem como observada a distribuição gráfica dos resíduos padronizados.

Antes de os dados serem submetidos à análise de variância, eles foram analisados quanto à homocedasticidade, pelo teste de Cochran (SNEDECOR e COCHRAN, 1989), e distribuição normal dos resíduos, pelo teste de Lilliefors (CAMPOS, 1979).

As variáveis dendrométricas do sabiá foram analisadas por intervalo de confiança ( $\alpha=0,05)$, por estarem presentes apenas nas parcelas do plantio consorciado, inviabilizando-se a análise de variância.

\section{RESULTADOS E DISCUSSÃO}

A menor sobrevivência, no plantio puro, foi observada em E. pellita, não diferenciando estatisticamente de E. tereticornis e E. robusta. No plantio consorciado, a menor sobrevivência foi apresentada pelo E. robusta, não se diferenciando estatisticamente de E. tereticornis e E. pellita. As plantas de E. camaldulensis apresentaram maior sobrevivência nos dois sistemas de plantio, e no plantio puro não diferenciaram estatisticamente de $E$. tereticornis e E. robusta. O sistema de plantio consorciado resultou em menor sobrevivência de plantas de E. robusta (Quadro 1).

Observando a sobrevivência das espécies de eucalipto, em outros sítios constata-se que a mortalidade ocorrida na cava deste estudo não difere muito do que tem sido encontrado em outros locais. Em áreas afetadas por excesso de sais e em cavas, a porcentagem de sobrevivência encontrada, nas diferentes espécies, variou de 68 a 99,31\% (SUN e DICKINSON, 1995; BENYON et al., 1999; SANTIAGO, 2000; SCHIAVO, 2004; TOMAR et al., 2003; MAHMOOD et al., 2003) e, em áreas não salinas, de 96 a 100\% (COUTINHO et al., 2004; DRUMOND et al., 1998).
Contrariando os resultados encontrados neste trabalho, plantas de E. camaldulensis, E. tereticornis e E. pellita, consorciados com Sesbania virgata (SANTIAGO, 2005) e E. camaldulensis consorciado com Acacia mangium (SCHIAVO, 2005), apresentaram maior sobrevivência em plantios puros.

Quadro 1 - Sobrevivência (\%) de plantas de quatro espécies de eucaliptos em plantios puros e consorciados com sabiá, 24 meses após o plantio

Table 1 - Plant survival (\%) of four eucalyptus species in pure stand or mixed with sabiá, 24 months after planting

\begin{tabular}{lcc}
\hline & Consórcio & Puro \\
\hline E. camaldulensis & $98,6 \mathrm{aA}$ & $91,7 \mathrm{aA}$ \\
E. pellita & $80,2 \mathrm{bA}$ & $73,6 \mathrm{bA}$ \\
E. tereticornis & $86,1 \mathrm{bA}$ & $84,0 \mathrm{abA}$ \\
E. robusta & $75,0 \mathrm{bB}$ & $84,0 \mathrm{abA}$ \\
\hline CV\% & \multicolumn{3}{c}{6,96} \\
\hline
\end{tabular}

Médias seguidas por letras maiúsculas iguais nas linhas e minúsculas nas colunas não diferem estatisticamente entre si, pelo teste de Tukey $(a=0,05) . C V \%=$ coeficiente de variação da análise de variância.

Com relação ao E. robusta, semelhantemente ao constatado neste estudo, Parrotta (1999) e Santiago (2005) observaram menor sobrevivência desta espécie quando consorciada com leguminosas arbóreas. Esses resultados levam a supor que E. robusta mostra-se mais sensível à competição quando consorciado com outras espécies.

O consórcio do $E$. robusta com sabiá, no sistema de manejo e no espaçamento adotados neste estudo, não foi adequado para a revegetação de cavas de extração de argila, por proporcionar maior mortalidade das plantas do que o sistema puro (Quadro 1). Dessa maneira, $E$. robusta parece apresentar maior competição interespecífica do que intra-específica, ficando evidente o efeito negativo do sabiá sobre a sobrevivência dessa espécie.

A área média de copa, ocupada por planta, não diferiu entre as espécies de eucalipto e entre os sistemas de plantio, aos 12 meses $(\mathrm{CV}=32,7 \%)$. As médias da área ocupada por planta, nessa ocasião, apresentaram os valores de $1,7 \mathrm{~m}^{2}$ (E. camaldulensis), 1,5 $\mathrm{m}^{2}$ (E. pellita) e $1,8 \mathrm{~m}^{2}$ (E. tereticornis e E. robusta).

O incremento médio mensal em DAS nas plantas de eucalipto não diferiu entre as espécies e não foi 
influenciado pelo sistema de plantio no período avaliado, apresentando comportamento quadrático em função do tempo, conforme mostrado na Figura 1.
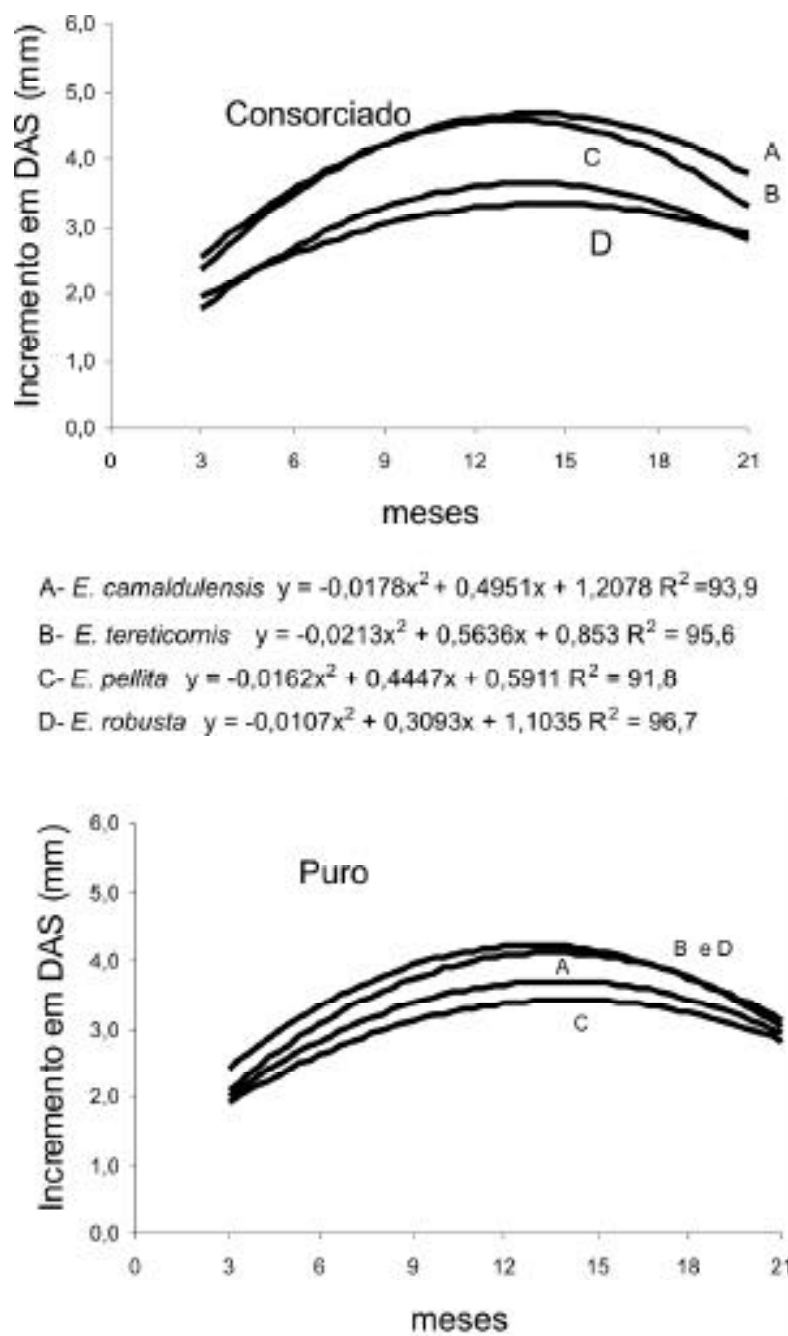

A- E. camaidulensis $y=-0,0148 x^{2}+0,4085 x+0,8982 R^{2}=96,5$

B- E. tereticarnis $y=-0,0185 x^{2}+0,4784 x+1,1273 R^{2}=92,7$

C-E. peilite $y=-0,0126 x^{2}+0,3528 x+0,9481 R^{2}=97,6$

D- E. robusta $y=-0,0184 x^{2}+0,4989 x+0,7411 R^{2}=98,1$

Figura 1 - Incremento médio mensal do diâmetro ao nível do solo (DAS) das espécies de eucalipto, nos sistemas de plantios puros e consorciados com sabiá, até 24 meses após o plantio $(\mathrm{CV} \%=10,4)$.

Figure 1 - Monthly average increase in diameter at ground level of the eucalyptus species, in the systems of pure stand and mixed with sabiá, 24 months after planting $(C V \%=10.4)$.
$\mathrm{O}$ incremento em altura não foi influenciado pelo sistema de plantio, embora as plantas de $E$. camaldulensis tenham apresentado maior incremento em altura $\left(24,1 \mathrm{~cm} \mathrm{mês}^{-1}\right)$ e as de E. pellita, menor $\left(15,8 \mathrm{~cm} \mathrm{mês}{ }^{-1}\right)$. O incremento em altura, em função do período, apresentou comportamento quadrático, conforme se observa na Figura 2.

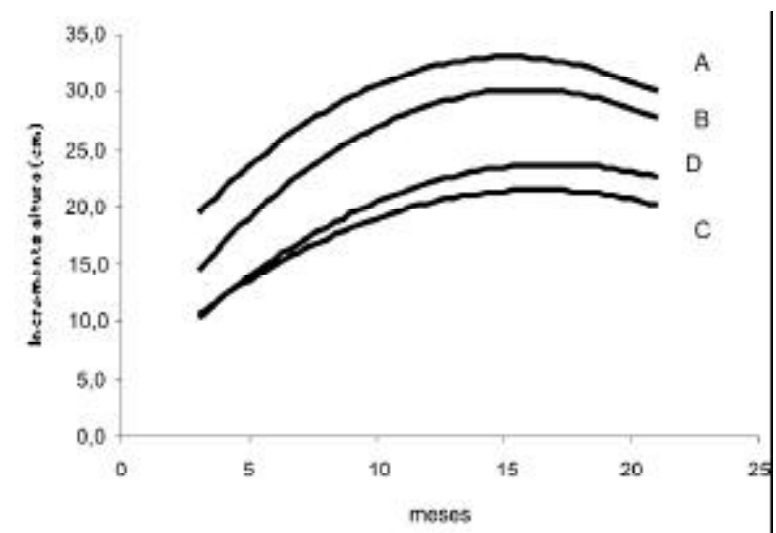

A- E. camaidulensis $y=-0,0922 x^{2}+2,7984 x+11,848 R^{2}=97,8$

B- E. tereticomis $y=-0,0943 x^{2}+2,9945 x+6,4353 R^{2}=99,5$

C- E. pollita $y=-0,061 x^{2}+1,9948 x+5,1453 R^{2}=99,6$

D- $E$ robusta $y=-0,0696 x^{2}+2,3499 x+3,8652 R^{2}=99,4$

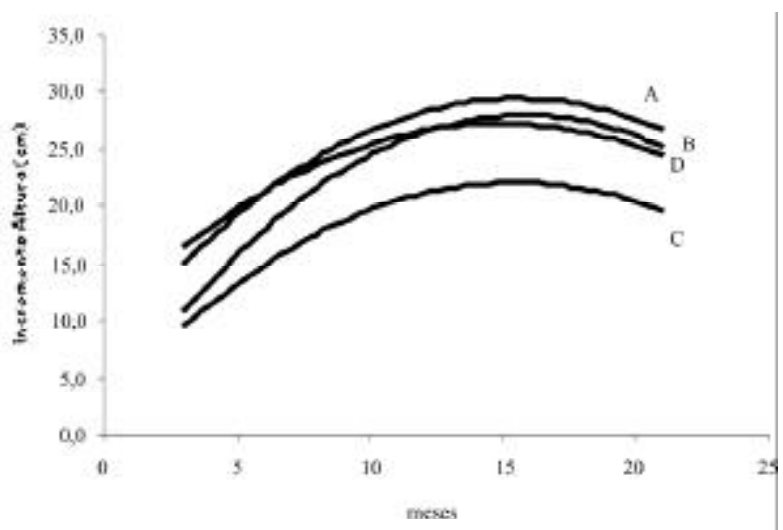

$$
\begin{aligned}
& \text { A- E. camaldulensis } y=-0,0916 x^{2}+2,8521 x+7,293 R^{2}=98,3 \\
& \text { B- E. tereticornis } y=-0,0769 x^{2}+2,2903 x+10,257 R^{2}=99,5 \\
& \text { C- E. pellita } y=-0,0809 x^{2}+2,5016 x+2,7335 R^{2}=98,2 \\
& \text { D- E. robusta } y=-0,1044 x^{2}+3,3058 x+1,8573 R^{2}=99,1
\end{aligned}
$$

Figura 2 - Incremento em altura das plantas de eucalipto, nos sistemas de plantios puros e consorciados, até 24 meses após o plantio $(\mathrm{CV} \%=8,1)$.

Figure 2 -Increase in height of eucalyptus plants, in pure stands and mixed systems, 24 months after planting $(C V \%=8.1)$.

R. Árvore, Viçosa-MG, v.32, n.3, p.395-405, 2008 
Apesar de o sistema de plantio não ter afetado significativamente o incremento em altura, é importante ressaltar que o incremento máximo no plantio consorciado foi atingido, aproximadamente, um mês após o sistema de plantio puro, com exceção do E. camaldulensis (Quadro 2). Esse resultado pode ser um indicativo de que no plantio consorciado as plantas de eucalipto demoram mais para serem influenciadas pela competição.

Aos 24 meses após o plantio, o DAP e o DAS não diferiram entre as espécies e nem entre os sistemas de plantio. A altura não respondeu ao sistema de plantio, e as plantas de E. pellita apresentaram menor altura média (Quadro 3). É importante ressaltar que os valores de altura e diâmetro encontrados neste trabalho são compatíveis com outros estudos conduzidos com várias espécies de eucalipto em distintas idades, sítios e solos, afetados ou não por excesso de sal (DRUMMOND et al., 1998; PARROTA, 1999; TOMAR et al., 2003; MAHMOOD et al., 2003).

Das espécies investigadas, E. pellita parece a menos indicada para o plantio em cavas de extração de argila, uma vez que apresentou menor sobrevivência no plantio puro, menor incremento em altura no período avaliado e menor altura média, aos 24 meses. Esses resultados podem estar relacionados à baixa resistência dessa espécie ao estresse salino, uma vez que o solo da cava em estudo é afetado por excesso de sais. Estudo realizado por Mendonça (2006), em casa de vegetação, a respeito do efeito da salinidade sobre aspectos fisiológicos de mudas de quatro espécies de eucalipto indicou que E. pellita foi sensível à salinidade ao apresentar redução na capacidade fotossintética em resposta à salinização do substrato, resultados que corroboram os encontrados neste estudo.

O sistema consorciado não influenciou o crescimento em altura e diâmetro durante o período de avaliação desta pesquisa (24 meses), entretanto, em resultados apresentados por outros estudos sobre sistemas consorciados (PARROTTA, 1999; NICHOLS et al., 2001; FORRESTER et al., 2004; BALIEIRO et al., 2004), observou-se que o efeito do consórcio sobre o crescimento das plantas começa a se revelar a partir do terceiro ano de plantio. Resultados contraditórios foram apresentados por Santiago (2005), que, avaliando quatro espécies de eucalipto em plantios puros e consorciados com $S$. virgata, verificou aos 10 meses, que o plantio puro resultou em maior DAS para o eucalipto, em comparação com o consorciado. Todavia, é importante ressaltar que, nesse estudo de Santiago (2005), o plantio do eucalipto foi realizado quando as plantas de sesbânia já estavam com um ano, o que pode ter favorecido a resposta do efeito do consórcio antes do terceiro ano.

Quadro 2 - Época de máximo, incremento em altura e diâmetro ao nível do solo (pontos de máximos), em meses, das espécies de eucalipto nos dois sistemas de plantio

Table 2 - Time of maximum increase in height and diameter at ground level (points of maxima), in months, for eucalyptus species in the two cropping systems

\begin{tabular}{lcccc}
\hline & & DAS & & Altura \\
\hline Espécie & Consorciado & Puro & Consorciado & Puro \\
E. camaldulensis & 13,9 & 13,8 & 14,9 & 15,6 \\
E. pellita & 13,2 & 12,9 & 15,9 & 14,9 \\
E. robusta & 13,7 & 14,0 & 16,4 & 15,5 \\
E. tereticornis & 14,5 & 13,6 & 16,7 & 15,8 \\
\hline
\end{tabular}

Quadro 3 - Características dendrométricas das espécies de eucalipto em plantios puros e consorciados com sabiá, aos 24 meses após o plantio

Table 3 - Dendrometric characteristics of eucalyptus species in pure stand or mixed with sabiá, 24 months after planting

\begin{tabular}{|c|c|c|c|c|c|c|c|c|c|}
\hline & \multicolumn{3}{|c|}{$\mathrm{DAS}(\mathrm{mm})$} & \multicolumn{3}{|c|}{$\mathrm{DAP}(\mathrm{mm})$} & \multicolumn{3}{|c|}{ Altura $(\mathrm{cm})$} \\
\hline Espécie & Consórcio & Puro & $\begin{array}{c}\text { Média } \\
\text { geral }\end{array}$ & Consórcio & Puro & Média geral & Consórcio & Puro & $\begin{array}{c}\text { Média } \\
\text { geral }\end{array}$ \\
\hline E. camaldulensis & $82,8 \mathrm{aA}$ & $64,7 \mathrm{aA}$ & $73,7 \mathbf{a}$ & $58,7 \mathrm{aA}$ & $44,6 \mathrm{aA}$ & 51,7 a & $661,2 \mathrm{aA}$ & $592,9 \mathrm{aA}$ & $627,1 \mathrm{a}$ \\
\hline E. pellita & $63,7 \mathrm{aA}$ & $61,8 \mathrm{aA}$ & $62,8 \mathbf{a}$ & $43,2 \mathrm{aA}$ & $40,3 \mathrm{aA}$ & $41,7 \mathrm{a}$ & $449,6 \mathrm{bA}$ & $435,7 \mathrm{bA}$ & $442,7 \mathrm{~b}$ \\
\hline E. tereticornis & $72,4 \mathrm{aA}$ & $66,7 \mathrm{aA}$ & $69,6 \mathbf{a}$ & $52,4 \mathrm{aA}$ & $45,1 \mathrm{aA}$ & $48,7 a$ & $612,2 \mathrm{abA}$ & $540,5 \mathrm{abA}$ & $576,4 \mathrm{ab}$ \\
\hline E. robusta & $63,0 \mathrm{aA}$ & $68,0 \mathrm{aA}$ & $65,5 \mathbf{a}$ & $43,4 \mathrm{aA}$ & $47,6 \mathrm{aA}$ & $45,5 a$ & $496,3 \mathrm{abA}$ & $555,4 \mathrm{abA}$ & 525,9 ab \\
\hline CV\% & \multicolumn{3}{|c|}{19,0} & \multicolumn{3}{|c|}{23,2} & \multicolumn{3}{|c|}{16,4} \\
\hline
\end{tabular}


A razão entre altura e DAP é utilizada para indicar o grau de competição entre plantas (GRONINGER et al., 1997). Espera-se uma menor razão altura/DAP, em condição de menor competição por luz. A razão altura/DAP do eucalipto não diferiu entre as espécies e não foi influenciada pelo sistema de plantio, aos 24 meses (Figura 3), indicando que até esse período o eucalipto não foi afetado pela competição por luz.

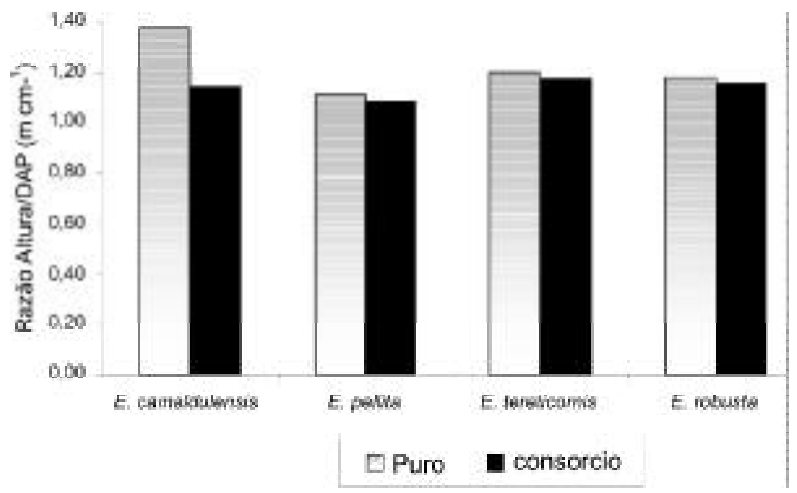

Figura 3 - Razão entre altura e DAP (m cm-1), aos 24 meses, das espécies de eucalipto em plantios puros e consorciados com sabiá $(\mathrm{CV} \%=11,3)$.

Figure 3-Height/BHD ratio ( $\mathrm{m} \mathrm{cm}-1)$ at 24 months for eucalyptus species in pure stands and mixed with sabiá $(C V \%=11.3)$.

Com relação à área e ao comprimento de raízes finas das espécies de eucalipto, pode-se observar que as plantas de E. camaldulensis e E. tereticornis apresentaram maiores valores dessas características, no plantio puro em relação ao consórcio com sabiá. No plantio puro, a maior área e o maior comprimento de raízes foram observados em E. camaldulensis, enquanto, no consorciado, E. robusta (Quadro 4).
Schmid e Kazda (2002) acreditaram que alta densidade de raízes finas indica melhor capacidade de obtenção de recursos, o que representa alta habilidade competitiva. Entretanto, neste estudo observou-se que, no plantio consorciado, a área e comprimento de raízes foram maiores em $E$. robusta, porém essa espécie apresentou redução na sobrevivência em decorrência da competição com sabiá (Quadro1). Inferese, assim, que a maior área e o maior comprimento de raízes finas não garantiram habilidade competitiva nas plantas dessa espécie. A habilidade competitiva, nesse caso, não pareceu relacionada à maior produção de raízes finas. A menor disponibilidade de água e nutrientes, normalmente, estimula a produção dessas raízes (GONÇALVEZ, 1995; KEITH et al., 1997; MISRA et al., 1998; GIARDINA e RYAN, 2002; SCHMID e KAZDA, 2002). Assim, a competição por água e nutrientes, imposta pelo sabiá sobre as plantas de eucalipto, pode ter promovido a maior produção de raízes finas.

A disponibilidade de água e nutrientes do solo influencia a distribuição do carbono entre os órgãos da planta. Aumento na disponibilidade de recursos reduz a alocação de carbono nas raízes (KEITH et al., 1997; GIARDINA e RYAN, 2002). De acordo com Misra et al. (1998), os fotoassimilados são, preferencialmente, utilizados pela raiz quando há limitações hídrica e nutricional. Em condição de estresse, as plantas são estimuladas a investir na produção de raízes finas de crescimento lento, favorecendo a seletividade. A biomassa de raízes finas em povoamento dePseudotsuga mensiessi (KEYES e GRIER, 1981), e de Picea abies (SCHMID e KAZDA, 2002) foi maior em sítios com baixa disponibilidade de nutrientes. Gonçalvez (1995) constatou que plantas de $E$. grandis apresentaram maior densidade de raízes finas em sítios menos férteis.

Quadro 4 - Área e comprimento de raízes finas das espécies de eucalipto nos dois sistemas de plantio, 24 meses após o plantio

Table 4 - Area and length of fine roots of eucalyptus species in the two cropping systems, 24 months after planting

\begin{tabular}{lcccc}
\hline & \multicolumn{2}{c}{ Área $\left(\mathrm{cm}^{2} \mathrm{dm}^{3}\right)$} & \multicolumn{2}{c}{ Comprimento $\left.\left(\mathrm{cm}^{3}\right)^{3}\right)$} \\
\hline Espécie & Consorciado & Puro & Consorciado & Puro \\
\hline E. camaldulensis & $21,2 \mathrm{bcB}$ & $37,4 \mathrm{aA}$ & $738,8 \mathrm{bcB}$ & $950,5 \mathrm{bA}$ \\
E. pellita & $27,2 \mathrm{bA}$ & $27,1 \mathrm{bA}$ & $603,6 \mathrm{cB}$ & $944,3 \mathrm{bA}$ \\
E. tereticornis & $15,0 \mathrm{cB}$ & $35,3 \mathrm{abA}$ & $1351,6 \mathrm{aA}$ & $1251,0 \mathrm{abA}$ \\
E. robusta & $41,8 \mathrm{aA}$ & $28,8 \mathrm{abA}$ & & 30,05 \\
\hline CV\% & & & & $1006,5 \mathrm{bA}$ \\
\hline
\end{tabular}


A menor área e o menor comprimento de raízes de E. camaldulensis e E. tereticornis no plantio consorciado, em comparação com o plantio puro, podem ser devidos nas melhores condições locais proporcionadas pelos benefícios desse sistema de plantio. O material vegetal proveniente da poda do sabiá pode ter resultado em melhorias nas condições do substrato, como maior manutenção da umidade e fornecimento de nutrientes, principalmente nitrogênio. Sabiá é eficiente em estabelecer simbiose com rizóbios e fungos micorrízicos (PRALON e MARTINS, 2001), apresentando biomassa aérea rica em nutrientes e com elevada velocidade de decomposição (ANDRADE et al., 2000), tendo potencial para aumentar a quantidade de carbono e nitrogênio no solo (COSTA et al., 1997). É importante ressaltar que estudos evidenciam que a maior disponibilidade de nitrogênio geralmente resulta em menor produção de raízes finas (MISRA et al., 1998; RAICH, 1998; McCONNAUGHAY e COLEMAN, 1999; BOUKCIM et al., 2005; LINKER e JOHNSON-RUTZKE, 2005).

Evidências de menor produção de raízes finas em plantio consorciado, em relação a plantios puros, também foram observadas em Picea abies com Fagus silvatica (SCHMID e KAZDA, 2002); emE. globulus com Acacia mearsnssi (FORRESTER et al., 2005) eE. camaldulensis consorciado com $S$. virgata (SANTIAGO, 2005).

As características biométricas, os intervalos de confiança em altura e diâmetro ao nível do solo e o porcentual médio de sobrevivência 24 meses após o plantio, das plantas de sabiá, quando consorciadas com as espécies de eucalipto, estão apresentadas no Quadro 5.

Paulino et al. (2002), avaliando o plantio puro de sabiá, em uma pastagem degradada, constataram elevado porcentual de sobrevivência (87\%) e encontraram uma altura média de 3,26 m. Os valores médios obtidos de sobrevivência e altura foram próximos e superiores aos verificados pelos autores anteriormente citados, indicando que o consórcio, até 24 meses, não prejudicou o crescimento do sabiá. Mendonça (2006) avaliou o crescimento e produção de raízes finas e de biomassa aérea de plantas de sabiá, em plantios puros e consorciados com eucalipto, observando que o consórcio não afetou a sobrevivência e altura das plantas, mas, aos 24 meses, o DAS foi superior no plantio puro.

\section{CONCLUSÕES}

Com base nos dados de sobrevivência e de crescimento das espécies de eucalipto até 24 meses de idade, pode-se concluir que E. pellita não é indicado para plantios em cavas de extração de argila na região Norte fluminense.

Não foi detectado, até o momento, efeito do sistema de plantio sobre o crescimento das espécies de eucalipto.

O consórcio com sabiá resultou em efeito negativo sobre a sobrevivência de E. robusta, sendo o consórcio entre essas duas espécies, no sistema de manejo e espaçamento adotados, não indicado para cava de extração de argila na região Norte fluminense.

\section{REFERÊNCIAS}

ANDRADE, A. G.; COSTA, G. S.; FARIA, S. M. Deposição e decomposição da serrapilheira em povoamentos de Mimosa caesalpiniaefolia, Acacia mangium e Acacia holosericea com quatro anos de idade em plantossolo. Revista Brasileira de Ciência do Solo, v.24, n.4, p.777-785, 2000.

Quadro 5 - Diâmetro ao nível do solo (DAS), em mm, altura (cm) e porcentual de sobrevivência (\%S) de plantas de sabiá consorciadas com eucalipto, aos 24 meses após o plantio

Table 5 - Diameter at ground level, in $\mathrm{mm}$, height $(\mathrm{cm})$ and percentage of survival $(\% \mathrm{~S})$ of sabiá mixed with eucalyptus, 24 months after planting

\begin{tabular}{|c|c|c|c|c|c|c|c|c|c|c|c|c|}
\hline \multirow[b]{3}{*}{ Parâmetros } & \multicolumn{12}{|c|}{ Espécies Consorciadas com Sabiá } \\
\hline & \multicolumn{3}{|c|}{ E. camaldulensis } & \multicolumn{3}{|c|}{ E. tereticornis } & \multicolumn{3}{|c|}{ E. pellita } & \multicolumn{3}{|c|}{ E. robusta } \\
\hline & Altura & DAS & $\mathrm{S} \%$ & Altura & DAS & $\mathrm{S} \%$ & Altura & DAS & $\mathrm{S} \%$ & Altura & DAS & S\% \\
\hline Média & 390,1 & 66,0 & 97,2 & 388,6 & 61,0 & 95,8 & 366,3 & 58,0 & 100,0 & 384,3 & 62,2 & 97,2 \\
\hline EPM & 10,0 & 1,9 & 2,8 & 8,6 & 1,6 & 2,7 & 11,3 & 1,9 & 0,0 & 9,7 & 1,7 & 2,8 \\
\hline $\mathrm{CV} \%$ & 21,5 & 24,0 & 5,7 & 18,4 & 22,3 & 5,6 & 26,1 & 28,2 & 0,0 & 18,6 & 22,7 & 5,7 \\
\hline $\mathrm{t}(\mathrm{a}=0,05)$ & 2,0 & 2,0 & - & 2,0 & 2,0 & - & 2,0 & 2,0 & - & 2,0 & 2,0 & - \\
\hline LI & 370,4 & 62,3 & - & 371,4 & 57,7 & - & 343,9 & 54,2 & - & 365,2 & 58,9 & - \\
\hline LS & 409,9 & 69,8 & - & 405,7 & 64,2 & - & 388,6 & 61,8 & - & 403,3 & 65,5 & - \\
\hline
\end{tabular}

R. Árvore, Viçosa-MG, v.32, n.3, p.395-405, 2008 
BALIEIRO, F. C.; FRANCO, A. A.; PEREIRA, M. G. Contribution of litter and nitrogen to soil under Pseudosamanea guachapele and Eucalyptus grandis plantations. Pesquisa Agropecuária Brasileira, v.39, n. 6, p.597-601, 2004.

BANZATTO, D. A.; KRONKA, S. N. Experimentação agrícola. Jaboticabal: FUNEP, 1992. 247p.

BAUHUS, J.; KHANNA, P. K.; MENDEN, N. Aboveground and belowground interactions in mixed plantations of Eucalyptus globulus and Acacia mearnsii. Canadian Journal of Forest Research, v.30, n.12, p.1886-1894, 2000.

BENYON, R. G. et al. Growth and water use of Eucalyptus camaldulensis and E. occidentallis on saline discharge site near Wellington, NSW, Australia. Agricultural Water Management, v.39, n.1-2, p.229-244, 1999.

BINKLEY, D. et al. Twenty years of stand of Eucalyptus saligna and nitrogen-fixing Facaltaria moluccana. Forest Ecology and Management, v.182, n.1-3, p.93-102, 2003.

BOUKCIM, H.; PAGES, L.; MOUSAIN, D. Local $\mathrm{NO}^{-3}$ or $\mathrm{NH}_{4}^{+}$supply modifies the root system architecture of Cedrus atlantica seedlings grown in a split root device. Journal of Plant Physiology, v.163, n.12, p.1293-1304, 2006.

CAMPOS, H. Estatística experimental não paramétrica. 3.ed. Piracicaba: Escola Superior de Agricultura Luiz de Queiroz, 1979.373p.

CHATTERJU, S.; PRICE, B. Regression analysis by example. New York: John Wiley \& Sons, 1977. 228p.

COSTA, G. S.; ANDRADE, A. G.; FARIA, S. M. Aporte de nutrientes pela serrapilheira deMimosa caesalpiniaefolia (sabiá) com seis anos de idade. In: SIMPÓSIONACIONALDERECUPERAÇÃODE ÁREAS DEGRADADAS, 3., Ouro Preto, 1997Anais... Viçosa-MG: Folha Florestal, 1997.p.344-334.

COUTINHO, J. L. B. et al. Avaliação do comportamento de espécies de Eucalyptus spp na Zona da Mata Pernambucana. I: Resultados do primeiro ano-2001. Revista Árvore, v.28, n.6, p.771-775, 2004.
DRUMOND, M. A.; OLIVEIRA, V. R.; CARVALHO, O. M. Comportamento silvicultural de espécies e procedências de Eucalyptus na região dos tabuleiros costeiros do estado do Sergipe. Revista Árvore, v.22, n.1, p.137-142, 1998.

FORRESTER, D. I.; BAUHUS, J.; COWIE, A. L. On the success and failure of mixed species tree plantations: lessons learned from a model system of Eucalyptus globulus and Acacia mearnsii. Forest Ecology and Management, v.209, n.1-2, p.147-155, 2005.

FORRESTER, D. I.; BAUHUS, J.; KHANNA, P. K. Growth dynamics in a mixed-species plantation of Eucalyptus globulus and Acacia mearnsii.

Forest Ecology and Management, v.193, n.1-2, p.81-95, 2004.

GAMA-RODRIGUES, A. C. Ciclagem de nutrientes por espécies florestais em povoamentos puros e mistos, em solos tabuleiros da Bahia, Brasil. 1997. 107f.Tese (Doutorado em Solos e Nutrição de Plantas) - Universidade Federal de Viçosa, Viçosa, MG, 1997.

GIARDINA, C. P.; RYAN, M. G. Total belowground carbon allocation in a fast-growing Eucalyptus plantation estimate using a carbon balance approach. Ecosystems, v.5, n.5, p.487499, 2002.

GONÇALVEZ, J. L. M. Características do sistema radicular de absorção do Eucalyptus grandis sob diferentes condições edáficas: I Distribuição de raízes nas camadas de solo. In: CONGRESSO BRASILEIRO DE CIÊNCIA DO SOLO, 1995, Viçosa, MG Anais... Viçosa, MG: Sociedade Brasileira de Ciência do Solo, 1995. p.876-878.

GRONINGER, J. W.; ZEDATER, S. M.; FREDERICTSEN, T. S. Stand characteristics of inter-cropped loblolly pine and black locust. Forest Ecology and Management, v.91, n.2-3, p.221-227, 1997.

HUNT, M. A.; UNWIN, G. L.; BEADLE, C. L. Effects of naturally regenerated Acacia dealbata on the productivity of a Eucalyptus nitens plantation in Tasmania, Australia. Forest Ecology and Management, v.117, n.1-3, p.75-85, 1999.

R. Árvore, Viçosa-MG, v.32, n.3, p.395-405, 2008 
JESUS, R. M.; DIAS, G. B. N. Eucalyptus/ Leucaena mixture experiment - growth and yield. Série Técnica IPEF, n.39, p.41-46, 1988.

KEYES, M. R.; GRIER, C. C. above and belowground net production in 40 yers-old Douglas-fir stands on low and high productivity sites.

Canadian Journal of Forest Research, v.11, p.599-605.

KEITH, H.; RAISON, R. J.; JACOBSEN, K. L. Allocation of carbon in mature eucalipt forest and some effects of soil phosphorus availability. Plant and Soil, v.196, n.1, p.81-99, 1997.

KUMAR, B. M.; KUMAR, S. S.; FISHER, R. F. Intercropping Teak with Leucena increases tree growth and modifies soil characteristics.

Agroforest. System, v.42, n.1, p.81-89, 1998.

LINKER, R.; JOHNSON-RUTZKE, C. J. Modeling the effect of abrupt changes in nitrogen availability on lettuce growth, root-shoot partitioning and nitrate concentration. Agricultural Systems, v.86, n.2, p.166-189, 2005.

MAHMOOD, K. et al. Genetc variation in Eucalyptus camaldulensis Dehnh for growth and stem straightness in a provenance-family trial on saltland in Pakistan. Forest Ecology and Management, v.176, n.1-3, p.405-416, 2003.

MENDONÇA, A. V. R. Reabilitação de cavas de extração de argila e tolerância de espécies florestais a salinidade. 2006. Tese (Doutorado em Produção Vegetal) - Universidade Federal do Norte Fluminense, Campos dos Goytacazes, 2006.

McCONNAUGHAY, K. D. M.; COLEMAN, J. S. Biomass allocation in plants: Ontogeny or optimality? A test along three resource gradients. Ecology, v.80, n.8, p.2581-2593, 1999.

MISRA, R. K. et al. Below and above ground growth of Eucalyptus nitens in a young plantation. II Nitrogen and Phosphorus. Forest Ecology and Management, v.106, n.2-3, p.295-306, 1998.

NICHOLS, J. D. et al. Intercropping legume trees with native timber trees rapidly restores cover to eroded tropical pasture without fertilization.

Forest Ecology and Management, v.152, n.1-3, p.195-209, 2001.

R. Árvore, Viçosa-MG, v.32, n.3, p.395-405, 2008
PARROTTA, J. A. Productivity, nutrient cycling, and succession in single and mixed species plantations of Casuarina equisetifolia, Eucalyptus robusta, and Leucaena leucocephala in Puerto Rico. Forest Ecology and Management, v.124, n.1, 45-77, 1999.

PAULINO, G. M. et al. Estudo da contribuição de espécies florestais para a recuperação de terras degradadas por pastagem em Conceição de Macabu, RJ. In: SIMPÓSIO NACIONAL DE RECUPERAÇÃO DE ÁREAS DEGRADADAS, 5., 2002.Anais... Belo Horizonte, MG: SOBRADE, 2002. p.508-509.

PRALON, A. Z.; MARTINS, M. A. Utilização do resíduo industrial ferkal na produção de mudas de Mimosa caesalpiniaefolia em estéril de extração de argila inoculadas com fungos micorrízicos arbusculares e rizóbio. Revista Brasileira de Ciência do Solo, v.25, n.1, p.55-63, 2001.

RAICH, J. W. Aboveground productivity and soil respiration in three Hawaiian rain forests. Forest Ecology and Management, v.107, n.1-3, p.309-318, 1998.

RAMOS, I. S. et al. Dimensionamento da indústria cerâmica em Campos dos Goytacazes, RJ. In: CONGRESSOBRASILEIRODECERÂMICA, 47., 2003, João Pessoa. Anais... João Pessoa: 2003. CD ROM.

RAMOS, D. P.; MANZATTO, C. V. Estudos de solos para ordenamento do uso cerâmico das argilas da Baixada Campista, estado do Rio de Janeiro. Rio de Janeiro: Embrapa Solos, 2003. 19p. (Documento, 52)

SANTIAGO, A. R. Eucalipto em plantios puros e consorciados com sesbânia na reabilitação de cavas de extração de argila. 77f. 2005 Dissertação (Mestrado em Produção Vegetal) - Universidade Estadual do Norte Fluminense, Campos dos Goytacazes, 2005.

SCHMID, I.; KAZDA, M. Root distribution of Norway spruce in monospecific and mixed stands on different soils. Forest Ecology and Management, v.159, n.1-2, p.37-47, 2002.

SNEDECOR, W.; COCHRAN, W. G. Statistical methods. 8.ed. Ames: Iowa State University Press, 1989. 502p. 
SCHIAVO, J. A. Revegetação de áreas degradadas pela extração de argila, com espécies micorrizadas de Acacia mangium, Sesbania virgata e Eucalyptus camaldulensis. 2005. 117f. Tese (Doutorado em Produção Vegetal) - Universidade Estadual do Norte Fluminense, Campos dos Goytacazes, 2005.

SCHIAVO, J. A. et al. Comportamento deAcacia mangium Willd e Eucalyptus camaldulensis, em plantio puro e consorciado, em áreas degradadas pela extração de argila no Município de Campos dos Goytacazes. FERTIBIO 2004. Lages: 2004. CD-ROM.

SUN, D.; DICKINSON, G. R. Salinity effects on tree growth, root distribuition and transpiration of Casuarina cunninghamiana and Eucalyptus camaldulensis planted on a saline site in tropical north Australia. Forest Ecology and Management, v.77, n.1-3, p.127-138, 1995.
TOMAR, O. S. et al. Performace of 31 tree species and soil conditions in plantation established with saline irrigation. Forest Ecology and

Management, v.177, n.1-3, p.333-346, 2003.

VALICHESKI, R. R. Avaliação técnica e econômica da reutilizaçao de áreas de extração de argila em Campos dos Goytacazes-RJ. 2004. 132f. Dissertação (Mestrado em Produção Vegetal) - Universidade Estadual do Norte Fluminense, Campos dos Goytacazes, 2004.

VEZZANI, F. M.; TEDESCO, M. J.; BARROS, N. F. Alterações dos nutrientes no solo e plantas em consórcio de eucalipto e Acácia negra. Revista Brasileira de Ciência do Solo, v.25, n.1, p.225-231, 2001. 